Published in final edited form as:

Ann Behav Med. 2017 February ; 51(1): 94-104. doi:10.1007/s12160-016-9831-7.

\title{
Perceived Weight Discrimination and 10-Year Risk of Allostatic Load Among US Adults
}

\author{
Maya Vadiveloo, PhD, $\mathbf{R D}^{1}$ and Josiemer Mattei, PhD, $\mathbf{M P H}^{2}$ \\ ${ }^{1}$ Department of Nutrition and Food Sciences, University of Rhode Island, Kingston, RI 02881, \\ USA \\ 2 Department of Nutrition, Harvard T. H. Chan School of Public Health, 665 Huntington Ave, Bldg \\ 2, Boston, MA 02115, USA
}

\begin{abstract}
Background-Discrimination promotes multisystem physiological dysregulation termed allostatic load, which predicts morbidity and mortality. It remains unclear whether weight-related discrimination influences allostatic load.

Purpose-The aim of this study was to prospectively examine 10-year associations between weight discrimination, allostatic load, and its components among adults 25-75 years in the Midlife Development in the US Biomarker Substudy.

Methods-Participants with information on weight discrimination were analyzed ( $n=986)$. At both timepoints, participants self-reported the frequency of perceived weight discrimination across nine scenarios as "never/rarely" (scored as 0), "sometimes" (1), or "often" (2). The two scores were averaged and then dichotomized as "experienced" versus "not experienced" discrimination. High allostatic load was defined as having $\geq 3$ out of 7 dysregulated systems (cardiovascular, sympathetic/ parasympathetic nervous systems, hypothalamic pituitary axis, inflammatory, lipid/ metabolic, and glucose metabolism), which collectively included 24 biomarkers. Relative risks (RR) were estimated from multivariate models adjusted for sociodemographic and health characteristics, other forms of discrimination, and BMI.
\end{abstract}

Results-Over $41 \%$ of the sample had obesity, and $6 \%$ reported weight discrimination at followup. In multivariable-adjusted analyses, individuals who experienced (versus did not experience) weight discrimination had twice the risk of high allostatic load (RR, 2.07; $95 \%$ CI, $1.21 ; 3.55$ for baseline discrimination; 2.16, $95 \%$ CI, 1.39; 3.36 for long-term discrimination). Weight discrimination was associated with lipid/ metabolic dysregulation (1.56; $95 \%$ CI 1.02, 2.40),

Correspondence to: Josiemer Mattei.

Electronic supplementary material The online version of this article (doi:10.1007/s12160-016-9831-7) contains supplementary material, which is available to authorized users.

Compliance with Ethical Standards

Authors' Statement of Conflict of Interest and Adherence to Ethical Standards Authors Maya Vadiveloo and Josiemer Mattei declare that they have no conflict of interest. All procedures, including the informed consent process, were conducted in accordance with the ethical standards of the responsible committee on human experimentation (institutional and national) and with the Helsinki Declaration of 1975, as revised in 2000. 
glucose metabolism $(1.99 ; 95 \%$ CI 1.34, 2.95), and inflammation (1.76; $95 \%$ CI 1.22, 2.54), but no other systems.

Conclusions-Perceived weight discrimination doubles the 10-year risk of high allostatic load. Eliminating weight stigma may reduce physiological dysregulation, improving obesity-related morbidity and mortality.

\section{Keywords}

Obesity stigma; Weight discrimination; Allostatic load; Allostasis; Dysregulation; Weight stigma

\section{Introduction}

Obesity is the leading contributor to disability-adjusted life years in the USA, at least partly due to its adverse effects on multiple health outcomes [1]. It is well-established that obesity etiology is both complex and manifold [2], suggesting a need for integrated biopsychosocial and biomedical approaches to address it [3]. Despite this, predominant approaches to treat obesity continue to accentuate the role of the individual [4], unintentionally contributing to a cycle that further entrenches obesity and its related health consequences by promoting stigmatization of this condition $[3,4]$.

Research suggests that pervasive individually targeted health campaigns increase public prejudice toward individuals with obesity by increasing the perception of obesity as a lifestyle choice resulting from weakness of character [5]. A downward consequence of this perception is increased weight-related stigma and, often, discrimination [6,7]. Weight discrimination is the fourth most prevalent form of discrimination among adults, after age, sex, and race-based discrimination [8]. Between 1995 and 2006, the absolute prevalence of weight discrimination increased from 7 to $12 \%$ in the USA, representing a $66 \%$ increase in prevalence and exceeding the proportion that could be attributed to concomitant increases in obesity [9]. Despite the lay belief that weight stigma motivates positive behavioral change [10], most evidence demonstrates that weight shaming promotes poorer dietary and exercise practices and health care avoidance [11,12], akin to how experienced racism correlates with negative health outcomes like cancer risk [13, 14]. As such, weight discrimination may contribute to obesity $[3,15]$ by discouraging individuals from seeking treatment, reducing engagement with social support, or promoting disordered eating patterns, less healthful food choices, and emotional dysregulation [12, 16-21]. Furthermore, weight stigma has been directly linked to overeating and physical inactivity in randomized controlled trials [10], providing plausible mechanisms through which weight stigma promotes physiologic dysregulation.

Discrimination also affects chronic stress, which could subsequently promote adverse physiologic changes [3, 15, 22-24]. For example, weight stigma has been associated with higher glycemic parameters [24] and C-reactive protein (CRP) [15] in large, longitudinal studies. In studies where weight stigma was experimentally manipulated, greater stigmatization resulted in sustained cortisol secretion [22, 25]. These findings echo existing research on the effects of perceived discrimination on allostatic load [26, 27], suggesting that 
there may be a similar connection between $w$ eight $d$ iscrimin atio $n$ a nd ph ysiolo gic dysregulation.

Allostatic load refers to the cumulative adverse adaptation of multiple physiological systems (i.e., cardiovascular, sympathetic, parasympathetic, and metabolic) in response to chronic stressors, which has been more strongly associated with chronic disease morbidity and mortality than traditional risk markers [28, 29]. Although the operational definitions of allostatic load vary across studies [28], the allostatic load metric is considered a robust estimator of multi-system dysregulation in population studies [30]. While it is valuable to examine allostatic load as a composite score, examining dysregulation within the individual allostatic load systems can help identify underlying pathways through which the allostatic load response is manifested, according to the population's specific characteristics [30]. As such, we examined whether the chronic stress associated with weight discrimination impacts both allostatic load and seven individual systems used to define allostatic load to elucidate the underlying pathways through which weight discrimination promotes dysregulation.

We propose that weight-related discrimination triggers multisystem dysregulation that adversely affects other health outcomes (e.g., cardiovascular disease) beyond the effects of obesity alone (Fig. 1). Similar to the cyclic obesity/weight-based stigma (COBWEBS) model [3], weight-related discrimination is characterized as a stressor that triggers a downward cascade of unfavorable psychosocial and behavioral processes that ultimately result in poor biological outcomes across multiple systems (e.g., metabolic syndrome, cardiovascular disease) $[31,32]$. To test this hypothesis, we used unique data from the national survey of Midlife Development in the USA (MIDUS) study to prospectively examine the 10-year associations between perceived weight discrimination and allostatic load among adults ages $25-74$ years.

\section{Methods}

\section{Participants}

We used data from the MIDUS I (1995-1996), MIDUS II (2004-2006), and MIDUS Biomarker Substudy (2004-2009) to examine associations between perceived weight discrimination, allostatic load, and the individual systems comprising allostatic load. Detailed information about the study's sampling procedures has been previously published [33]. Briefly, 7108 non-institutionalized adults (including 950 siblings and 1914 twins) aged 25-74 years participated in a telephone survey conducted via random digit dialing in 19951996. At follow-up between 9 and 10 years later, approximately 4900 members of the original cohort responded to an additional phone survey; the mortality-adjusted longitudinal response rate at MIDUS II was $75 \%$. During the 10-year follow-up, a subset of 1255 adults who completed the phone interview and questionnaires was randomly selected and invited to participate in a biomarker substudy. The present analysis includes those in the biomarker substudy with sufficient information to compute allostatic load $(n=1233)$ or the individual allostatic load systems $(n=1158-1254)$ and who had information on perceived weight discrimination $(n=986)$. 


\section{Perceived Weight Discrimination}

Participants self-reported instances of perceived discrimination within interpersonal relationships on a day-to-day basis at both the baseline and 10-year follow-up surveys. Nine scenarios about interpersonal discrimination were queried with the question "How often on a day-to-day basis do you experience each of the following types of discrimination?" The scenarios included "you are treated with less courtesy than other people," "you are treated with less respect than other people," "you receive poorer service than other people at restaurants or stores," "people act as if they are afraid of you," "people act as if they think you are dishonest," "people act as if they think you are not as good as they are," "you are called names or insulted," and "you are threatened or harassed." The frequency categories for these scenarios included "often," "sometimes," "rarely," or "never." These questions were initially developed for a study examining racial discrimination and have been used widely since then [34].

Similar to Puhl and others [8], only participants reporting discrimination "sometimes" or "often" were counted as instances of discrimination. We constructed a continuous measure of perceived discrimination that allocated 2 points for every instance that a discrimination scenario was reported as "often," 1 point for every scenario reported as "sometimes," and 0 point for those who reported discrimination "rarely" or "never." Separately, participants were asked to select the primary reason(s) for discrimination, from among the following options: age, gender, race, height or weight, ethnicity or nationality, physical disability, some aspects of appearance other than weight or height, sexual orientation, religion, and other reasons. Like previous studies, we refer to the "height or weight" variable as "weight discrimination" throughout the manuscript [8].

We constructed two variables for perceived weight discrimination at both baseline and 10year follow-up. First, a continuous measure of perceived weight discrimination was computed from the continuous perceived discrimination score for individuals who reported "weight" as a primary reason for discrimination. The observed range for this score was 010 at baseline and 10-year follow-up. Secondly, a categorical indicator variable was created for individuals who experienced any vs. no perceived weight discrimination.

Individuals who reported no instances of discrimination received a weight discrimination score of $0(n=126)$. We also carried baseline values forward for non-responders at the 10 year follow-up who reported discrimination related to weight at baseline based on the correlation between these two measures $(n=215, r=0.40, p<0.0001)$. Individuals who refused to respond to the question or whose responses were deemed "inappropriate" by study administrators were coded as missing $(n=46)$. Complete information was available for 986 participants at baseline and 940 at follow-up. The two exposures of interest were baseline weight discrimination and long-term weight discrimination. Long-term discrimination was computed as the average value of perceived discrimination at baseline and at 10-year follow-up or as discrimination at 10 years for individuals with missing baseline data. 


\section{Allostatic Load}

Allostatic load was comprehensively measured and defined in accordance with previous studies conducted within this population using a score that captured dysregulation across seven systems, including the sympathetic and parasympathetic nervous systems, hypothalamic pituitary adrenal (HPA) axis, cardiovascular functioning, lipid and general metabolic activity, glucose metabolism, and inflammatory system [31, 35, 36] (Table 1). All physiologic measures were collected during the Biomarker Substudy visit, which corresponded with the timing of the 10-year follow-up exam.

Sympathetic nervous system functioning was measured with 12-h overnight urinary measurements of epinephrine and norepinephrine via high-pressure liquid chromatography, and levels were reported per level of creatinine ( $\mathrm{g}$ ). Parasympathetic nervous system activity was measured by four heart rate variability parameters during an 11-min seated rest period using an electrocardiograph: low frequency spectral power, high frequency spectral power, the standard deviation of heartbeat to heartbeat intervals, and the root mean square of successive differences. Overnight urinary cortisol and serum dehydroepiandrosterone sulfate (DHEA-S) were used as markers of HPA activity. Markers of cardiovascular functioning included resting systolic blood pressure (SBP), heart rate, and pulse pressure. Lipid/fat metabolism markers included high-density lipoprotein (HDL), low-density lipoprotein (LDL), triglycerides, body mass index (BMI), and waist-to-hip ratio (WHR). Glycosylated hemoglobin (HbA1c), fasting glucose, and the homeostasis model of insulin resistance (HOMA-IR) were used to assess glucose metabolism. Inflammation was measured with plasma CRP, fibrinogen, serum interleukin-6 (IL-6), the soluble adhesion molecule Eselectin, and intracellular adhesion molecule-1. All blood, urine, saliva, cardiovascular, and heart rate variability measurements were collected during an overnight stay at one of three University General Clinical Research Centers. Detailed collection protocols for each biomarker have been previously published [31,37]. Consistent with previous studies [36], we computed a system risk score for each of the seven systems that was in the upper or lower quartile of the biomarker population-specific distribution, based on whether high or low values of the parameter were generally associated with higher health risk. Additionally, consistent with previous research [35, 38-40], participants who reported using medications to treat dysregulated parameters were categorized as high risk for that parameter to account for pre-existing dysregulation. These medications included antihypertensive medications for high SBP; heart rate-reducing medications (e.g., beta-blockers and atrioventricular nodal blockers) for high resting heart rate; hypoglycemic agents for dysregulated fasting glucose and $\mathrm{HbA1c}$; statins, cholesterol absorption inhibitors, niacin and/or bile acid sequestrants for dysregulated LDL; fibrates for elevated serum triglycerides; testosterone for dysregulated DHEA-S; and anti-inflammatory medications (including non-steroidal anti-inflammatory medications) for dysregulated CRP and IL-6. The number of participants with dysregulated parameters including and excluding medication data in the definition of allostatic load is shown in Supplemental Table 1. In sensitivity analyses, we examined all associations excluding the use of medications in the definitions of system dysregulation.

System risk scores were continuous and computed by calculating the proportion of individual biomarkers within the system that were dysregulated. Scores could range from 0 
to 1 (corresponding with $0-100 \%$ of system biomarkers in high-risk range). We only computed system risk scores for participants with values for at least half of the system's biomarkers. Over $90 \%$ of participants had information for all seven systems, and most participants $(98 \%)$ had complete data for all systems excluding the parasympathetic measures; 106 participants (8\%) were missing information on the parasympathetic parameters due to instrumentation failures and/or measurement difficulties.

The allostatic load variable was computed for participants with data on at least six of the seven systems by summing the seven system risk scores; total allostatic load scores ranged from 0 to 7, with higher scores indicative of more dysregulation. An indicator variable for high versus low allostatic load was created: allostatic load summary scores $>3$ were considered "high" and scores $<3$ were considered "low." Although the median allostatic load score in this population was 2 , using a higher cut point allowed us to capture individuals at higher disease risk [39].

\section{Covariates}

Potential confounding variables were selected based on their relevance from prior literature. We used self-reported information collected during the follow-up period for these variables: age, race (white, black, other), household income ( $>\$ 100,000 /$ year), educational attainment ( $<$ high school, high school graduate, some college, college graduate, or more), smoking status (never, former, current), and physical activity (engagement in regular exercise at least 20 min three times per week). Baseline rather than follow-up values were used for BMI and for perceived discrimination related to age, race, and/or sex (the three most common forms of discrimination (8)) because prolonged stress is more strongly related to allostatic load [28]. These variables were computed in the same manner as weight discrimination.

\section{Statistical Methods}

Mixed linear models with maximum likelihood estimation and family membership as a random effect were used to examine the continuous associations between perceived weight discrimination and allostatic load. Family membership was incorporated as a random effect to account for clustering since the sample included participants from the sibling/twin subsamples of the main MIDUS study [31]. A generalized linear model procedure was used to estimate relative risks (RR) using Poisson regression with robust error variance [41], as this method produces $95 \%$ confidence intervals with the correct coverage. Base models were adjusted for age and sex. The first multivariable-adjusted model (model 1) further adjusted for race, household income, smoking status, and educational attainment. Model 2 further incorporated physical activity, and model 3 was further adjusted for baseline perceived race, sex, and age discrimination. Our final model (model 4) also adjusted for baseline BMI. We tested for the presence of interactions between perceived weight discrimination and sex, BMI, physical activity, smoking status, and race, sex, or age discrimination using a $p$ value $<0.05$ to establish significance. We also used Baron and Kenny criteria [42] to examine whether health behaviors like smoking and physical activity mediated the association between weight discrimination and allostatic load. Additionally, we examined the associations between perceived weight discrimination and individual system dysregulation in order to provide insight into the biological pathways underlying any 
observed associations. Finally, in sensitivity analyses, we excluded BMI and WHR from the definition of lipid/metabolic dysregulation in the calculation of allostatic load. All analyses were conducted with SAS v 9.4 (SAS Institute Inc., Cary, NC, USA).

\section{Results}

The study sample was predominately comprised of white (93\%), female (57\%), middleaged adults (mean age $=57$ years) with high educational attainment $(47 \%$ with a college education or higher) (Table 2). More than $75 \%$ of participants reported engaging in regular physical activity, $15 \%$ were current smokers, and more than $75 \%$ were classified with either overweight or obesity. At baseline, nearly $4 \%$ of participants reported experiencing weight-related discrimination, with an average discrimination score of 0.13 (0.76). At follow-up, this percentage increased to approximately $6 \%$ with average discrimination values of 0.22 (1.09). When medication was included in the definition of high allostatic load, $18 \%$ of participants met the criteria, while only $13 \%$ met the criteria when medication usage was excluded.

No significant interactions between perceived weight discrimination and relevant covariates were detected (data not shown). Results were similar regardless of whether we used medications to operationalize allostatic load; thus, those presented hereafter include medication information to capture already deregulated parameters. Compared to individuals who did not experience weight discrimination, both baseline and long-term perceived weight discrimination were associated with more than double the risk of high allostatic load in final multivariable models (RR, 2.07; $95 \%$ CI 1.21, 3.55 for baseline discrimination and RR, 2.16; $95 \%$ CI 1.39, 3.36 for long-term discrimination) (Table 3). Similar associations were observed when these associations were examined using the continuous weight discrimination score and allostatic load variables $(\beta=0.11, p=0.01$ for baseline discrimination and $\beta=0.19, p=0.0001$ for long-term discrimination). Additionally, the effect of weight discrimination on allostatic load was partly mediated ( $5 \%)$ through decreased physical activity among those who experienced versus did not experience weight discrimination (data not shown). Perceived race, sex, and age discrimination were not significantly associated with allostatic load in final models (data not shown). In sensitivity analyses, when BMI and WHR were excluded from the definition of allostatic load, baseline perceived weight discrimination was not significantly associated with allostatic load, but long-term weight discrimination remained associated with allostatic load after controlling for baseline BMI (RR, 1.62; $95 \%$ CI 1.01, 2.62; $\mathrm{p}=0.047)$.

Overall, compared to individuals reporting no weight-related discrimination, long-term weight discrimination was most strongly associated with metabolic/lipid dysregulation (RR, 1.56; $95 \%$ CI 1.02, 2.40), glucose metabolism (RR, 1.99; $95 \%$ CI 1.34, 2.95), and inflammatory parameters (RR, 1.76; $95 \%$ CI 1.22, 2.54) after adjustment for other confounding variables including baseline BMI (Table 4). Weight discrimination was not significantly associated with CVD function, sympathetic or parasympathetic nervous system dysregulation, or HPA dysfunction. 


\section{Discussion}

Perceived baseline and long-term weight discrimination were associated with more than twice the risk of high allostatic load in this sample. The detrimental effects of weight discrimination on allostatic load persisted following adjustment for BMI, suggesting that perceived weight-related discrimination adversely affects overall physiological regulation beyond what can be attributed to excess weight alone. Further support for the independent associations between weight discrimination and allostatic load was observed when BMI and WHR were excluded from the operationalization of allostatic load, and the long-term associations remained significant. When the individual systems comprising allostatic load were examined separately, perceived weight discrimination was most strongly associated with lipid/metabolic dysregulation, glucose metabolism, and markers of inflammation. Taken together, these results suggest that the stigma associated with having excess weight adversely influences allostatic load, and potentially chronic disease morbidity and mortality, highlighting a need for prevention efforts to reduce weight-related stigma in diverse settings.

While limited, empirical studies demonstrate that reducing weight-related stigma favorably affects weight-loss self-efficacy and attitudes toward exercise. In an experimental study, Pearl and Lebowitz demonstrated that overweight and obese participants who read passages that implicate the food environment vs. personal responsibility in obesity etiology had greater self-efficacy to lose weight and no increase in weight stigmatizing attitudes that adversely affect weight control [5]. Similarly, US women exposed to neutral vs. stereotypical images of a woman with obesity exercising had more favorable attitudes toward exercise engagement and lower weight-based stigma [43].

Our results suggest that perceiving weight discrimination can adversely affect multiple biological systems and are consistent with research examining individual biomarkers. In MIDUS, Tsenkova and others [24] noted that experiencing weight discrimination amplified the adverse effects of elevated WHR on HbA1c. Among community-dwelling adults with diabetes, researchers found that participants experienced worse glycemic outcomes if they had experienced weight-based discrimination [11]. Similar to the present study, the changes in glycemic markers persisted even after accounting for body weight and other forms of discrimination [11]. Moreover, the participants from the study conducted by Potter and others also reported worse diabetes self-care practices related to diet, exercise, and blood glucose monitoring, providing insight into the pathways by which weight discrimination adversely impacts physiologic parameters. These observed negative behavioral adaptations support the pathways proposed in our conceptual model relating weight discrimination to allostatic load. Another study noted that weight-related discrimination was associated with inflammatory markers like CRP among overweight but not obese individuals [15], and also suggested that worse self-care practices may underlie the associations between weightrelated discrimination and health outcomes. The significant findings in overweight rather than obese individuals implied that that weight discrimination may support the development and maintenance of obesity by activating inflammatory pathways [15].

Although allostatic load should primarily be evaluated as a matrix of dysregulated systems, investigating the individual systems informs our understanding of the biological 
underpinnings of an important risk marker. This study primarily implicated three of the seven systems in the association between weight discrimination and allostatic load, potentially identifying relevant treatment priorities. However, additional research into all systems remains necessary because the time course of metabolic dysregulation and the duration and mechanism of action of the biomarkers is not well understood. For example, it remains unclear whether obesity precedes HPA axis dysregulation or vice versa, and whether it results in hypo- or hyper-activity or volatility [44]. In the present study, many of the primary markers of HPA axis and CVD dysregulation associated with allostatic load were not affected by perceived weight discrimination, potentially suggesting that obesity precedes HPA axis dysregulation and induces some volatility [44]. However, because adrenal cortisol and adipose tissue cortisol may be differentially affected by obesity [44] and because biomarkers were only measured once during the follow-up period, we may have been unable to discern the critical window and/or site where HPA dysregulation would occur.

Experiencing weight discrimination appears to promote many of the pathologic features of obesity, such as inflammation, lipid/metabolic imbalances, glycemic dysregulation, and more holistically, allostatic load. Although the pathways through which weight discrimination influences allostatic load may be interconnected and multifactorial, this complexity provides promising opportunities for further research. It may be informative to investigate how discrimination relates to allostatic load parameters in more diverse populations where being overweight is less stigmatized, and whether factors like healthcare access can also modulate the effect of weight discrimination on health. While we did not detect any significant interactions between perceived weight discrimination and physical activity or smoking, and detected minimal mediation through physical activity, other research has found that health behaviors during adulthood partly explain the association between adverse events in early life and subsequent allostatic load [45]. For example, research in MIDUS has established a link between positive coping strategies and social support on allostatic load [36] that warrants additional exploration in individuals who experience weight discrimination. Physical activity also deserves further attention as it is possible that a more precise measure would more strongly mediate the association between weight discrimination and allostatic load.

In addition, while the associations between weight discrimination and allostatic load were robust in this study, 10 years of follow-up may provide only an indication of the potential full effect that weight discrimination could have on cumulative physiological dysregulation throughout longer periods of time or at different lifecycles. More longitudinal research with longer follow-up periods and repeated measurements would enhance our understanding of the time course of weight discrimination related to allostatic load development as well as critical windows when risk can be modified [28]. Finally, it may be important to establish confluence between clinical cut points and population-based cut points for the various biomarkers encompassing allostatic load to more accurately determine risk estimates.

Some limitations of the present analysis must be noted. Dietary information was not collected in the MIDUS study, which may be an important confounding or mediating variable in the association between perceived weight discrimination and allostatic loadparticularly because poor dietary choices have been related to the effects of discrimination 
on glycemic control [46]. Participant non-response rates on the questions about perceived discrimination also reduced the final sample size. Because non-response was higher among smoking, younger, women with lower self-reported physical activity, and higher BMI at baseline (data not shown), we expect that our risk estimates were attenuated and that the associations between weight discrimination and allostatic load are actually stronger than what we were able to observe. Finally, because participants could select multiple primary reasons for discrimination, it is possible that individuals who reported multiple forms of discrimination differed from individuals who only reported weight discrimination. However, associations did not change after controlling for other forms of reported discrimination, which improves the robustness of our findings.

The present study has several strengths that warrant mention. First, this study utilizes data from a large US national sample. Additionally, much research to date examining allostatic load have used limited markers or have been cross-sectional despite a call for more longitudinal research [28]; our study precisely measured multiple biomarkers across seven systems, and the nearly 10 years of follow-up provide important insight into the cumulative effects of weight discrimination as a stressor on multi-system dysregulation. By accounting for family relationships within the cohort, we reduced bias related to shared genetic or environmental factors that contribute to weight and metabolic dysregulation. The present study also builds upon existing evidence that self-reported weight discrimination adversely influences biochemical parameters beyond the effect of actual weight [11]. Given the established connection between personal responsibility campaigns and increased obesity stigma [5], the results from this study have important policy implications with respect to framing obesity prevention campaigns as well as treatment implications for clinicians working with clients with obesity.

The adverse health effects of obesity are well documented and require concerted efforts to treat. The emphasis on personal responsibility in the USA has had the effect of further stigmatizing obesity, resulting in less favorable health outcomes within this vulnerable population [4]. Weight discrimination was recently associated with a nearly $60 \%$ increase in overall mortality risk among MIDUS participants [47], and it is plausible that this hazard is at least partly mediated by allostatic load. The magnitude of risk observed between weight discrimination and allostatic load is greater than what has been observed for poor quality dietary patterns and allostatic load [40], and comparable to physical inactivity [48], drawing attention to weight discrimination as a significant allostatic load risk factor. Given that high allostatic load has been shown to be robustly associated with type 2 diabetes, hypertension, cardiovascular disease, and mortality [39, 49], targeted efforts to reduce weight discrimination are warranted. From a disease prevention standpoint, it is imperative to develop less stigmatizing public health campaigns and clinical approaches to reduce physiological dysregulation and long-term chronic disease risk among individuals with obesity or at risk for obesity. Simultaneously, directed efforts to better understand the pathways through which weight discrimination influences allostatic load can improve treatment targets and health outcomes among the substantial proportion of the population with weight-related comorbidities. 


\section{Supplementary Material}

Refer to Web version on PubMed Central for supplementary material.

\section{Acknowledgments}

This work was supported by the NHLBI (grant numbers HL49086, HL60692). Funding was also provided by a Mentored Career Development Award to Promote Faculty Diversity in Biomedical Research from the NHLBI (J.M., grant number K01-HL120951), and an NIH Ruth L Kirschstein Postdoctoral Fellowship (M.V., grant number 5 T32 DK 7703-19). NIH had no role in the design, analysis, or writing of this article.

\section{References}

1. Forouzanfar MH, Alexander L, Anderson HR, et al. Global, regional, and national comparative risk assessment of 79 behavioural, environmental and occupational, and metabolic risks or clusters of risks in 188 countries, 1990-2013: A systematic analysis for the Global Burden of Disease Study 2013. Lancet. 2015

2. Frood S, Johnston LM, Matteson CL, Finegood DT. Obesity, complexity, and the role of the health system. Curr Obes Rep. 2013; 2:320-326. [PubMed: 24273701]

3. Tomiyama AJ. Weight stigma is stressful. A review of evidence for the cyclic obesity/weight-based stigma model. Appetite. 2014; 82:8-15. [PubMed: 24997407]

4. Teixeira FV, Pais-Ribeiro JL, Maia AR. Beliefs and practices of healthcare providers regarding obesity: a systematic review. Rev Assoc Med Bras. 2012; 58:254-262. [PubMed: 22569623]

5. Pearl RL, Lebowitz MS. Beyond personal responsibility: effects of causal attributions for overweight and obesity on weight-related beliefs, stigma, and policy support. Psychol Health. 2014; 29:1176-1191. [PubMed: 24754230]

6. Goffman, E. Stigma: notes on the Management of Spoiled Identity. Prentice-Hall; Englewood Cliffs: 1963.

7. Allport, GW. The nature of prejudice. Addison-Wesley; Cambridge, MA: 1954.

8. Puhl RM, Andreyeva T, Brownell KD. Perceptions of weight discrimination: prevalence and comparison to race and gender discrimination in America. Int J Obes (Lond). 2008; 32:992-1000. [PubMed: 18317471]

9. Andreyeva T, Puhl RM, Brownell KD. Changes in perceived weight discrimination among Americans, 1995-1996 through 2004-2006. Obesity (Silver Spring). 2008; 16:1129-1134. [PubMed: 18356847]

10. Nolan LJ, Eshleman A. Paved with good intentions: paradoxical eating responses to weight stigma. Appetite. 2016; 102:15-24. [PubMed: 26802721]

11. Potter L, Wallston K, Trief P, et al. Attributing discrimination to weight: associations with wellbeing, self-care, and disease status in patients with type 2 diabetes mellitus. J Behav Med. 2015

12. Phelan SM, Burgess DJ, Yeazel MW, et al. Impact of weight bias and stigma on quality of care and outcomes for patients with obesity. Obes Rev. 2015; 16:319-326. [PubMed: 25752756]

13. Berger M, Sarnyai Z. "more than skin deep": stress neurobiology and mental health consequences of racial discrimination. Stress. 2015; 18:1-10. [PubMed: 25407297]

14. Black LL, Johnson R, VanHoose L. The relationship between perceived racism/discrimination and health among black American women: a review of the literature from 2003 to 2013. J Racial Ethn Health Disparities. 2015; 2:11-20. [PubMed: 25973361]

15. Sutin AR, Stephan Y, Luchetti M, Terracciano A. Perceived weight discrimination and C-reactive protein. Obesity. 2014; 22:1959-1961. [PubMed: 24828961]

16. Puhl RM, Brownell KD. Confronting and coping with weight stigma: an investigation of overweight and obese adults. Obesity (Silver Spring). 2006; 14:1802-1815. [PubMed: 17062811]

17. Haines J, Neumark-Sztainer D, Eisenberg ME, Hannan PJ. Weight teasing and disordered eating behaviors in adolescents: longitudinal findings from project EAT (eating among teens). Pediatrics. 2006; 117:e209-215. [PubMed: 16452330] 
18. Puhl RM, Moss-Racusin CA, Schwartz MB. Internalization of weight bias: implications for binge eating and emotional well-being. Obesity (Silver Spring). 2007; 15:19-23. [PubMed: 17228027]

19. Phelan SM, Burgess DJ, Puhl R, et al. The adverse effect of weight stigma on the well-being of medical students with overweight or obesity: findings from a National Survey. J Gen Intern Med. 2015; 30:1251-1258. [PubMed: 26173517]

20. Sikorski C, Luppa M, Luck T, Riedel-Heller SG. Weight stigma "gets under the skin"-evidence for an adapted psychological mediation framework: A systematic review. Obesity (Silver Spring). 2015; 23:266-276. [PubMed: 25627624]

21. Sutin A, Robinson E, Daly M, Terracciano A. Weight discrimination and unhealthy eating-related behaviors. Appetite. 2016; 102:83-89. [PubMed: 26877216]

22. Schvey NA, Puhl RM, Brownell KD. The stress of stigma: exploring the effect of weight stigma on cortisol reactivity. Psychosom Med. 2014; 76:156-162. [PubMed: 24434951]

23. Tomiyama AJ, Epel ES, McClatchey TM, et al. Associations of weight stigma with cortisol and oxidative stress independent of adiposity. Health Psychol. 2014; 33:862-867. [PubMed: 25068456]

24. Tsenkova VK, Carr D, Schoeller DA, Ryff CD. Perceived weight discrimination amplifies the link between central adiposity and non-diabetic glycemic control (HbA1c). Ann Behav Med. 2011; 41:243-251. [PubMed: 21136227]

25. Himmelstein MS, Incollingo Belsky AC, Tomiyama AJ. The weight of stigma: cortisol reactivity to manipulated weight stigma. Obesity (Silver Spring). 2015; 23:368-374. [PubMed: 25522347]

26. Upchurch DM, Stein J, Greendale GA, et al. A longitudinal investigation of race, socioeconomic status, and psychosocial mediators of allostatic load in midlife women: findings from the study of women's health across the nation. Psychosom Med. 2015; 77:402-412. [PubMed: 25886828]

27. Fuller-Rowell TE, Evans GW, Ong AD. Poverty and health: the mediating role of perceived discrimination. Psychol Sci. 2012; 23:734-739. [PubMed: 22700331]

28. Gallo LC, Fortmann AL, Mattei J. Allostatic load and the assessment of cumulative biological risk in biobehavioral medicine: challenges and opportunities. Psychosom Med. 2014; 76:478-480. [PubMed: 25141272]

29. Juster RP, McEwen BS, Lupien SJ. Allostatic load biomarkers of chronic stress and impact on health and cognition. Neurosci Biobehav Rev. 2010; 35:2-16. [PubMed: 19822172]

30. Howard JT, Sparks PJ. Does allostatic load calculation method matter? Evaluation of different methods and individual biomarkers functioning by race/ethnicity and educational level. American Journal of Human Biology. 2016 DOI: 10.1002/ajhb.22843.

31. Gruenewald TL, Karlamangla AS, Hu P, et al. History of socioeconomic disadvantage and allostatic load in later life. Social Science \& Medicine. 2012; 74:75-83. [PubMed: 22115943]

32. Papadopoulos S, Brennan L. Correlates of weight stigma in adults with overweight and obesity: a systematic literature review. Obesity (Silver Spring). 2015; 23:1743-1760. [PubMed: 26260279]

33. Radler BT, Ryff CD. Who participates? Accounting for longitudinal retention in the MIDUS National Study of health and well-being. Journal of Aging and Health. 2010; 22:307-331. [PubMed: 20103686]

34. Almeida DM. National Survey of Midlife Development in the United States (MIDUS I) National Study of Daily Experiences (NSDE), 1996-1997. Inter-university Consortium for Political and Social Research (ICPSR) [distributor]. 2015

35. Seeman M, Stein Merkin S, Karlamangla A, Koretz B, Seeman T. Social status and biological dysregulation: the "status syndrome" and allostatic load. Soc Sci Med. 2014; 118:143-151. [PubMed: 25112569]

36. Brooks KP, Gruenewald T, Karlamangla A, et al. Social relationships and allostatic load in the MIDUS study. Health Psychol. 2014; 33:1373-1381. [PubMed: 24447186]

37. Ryff CD, Seeman T, Weinstein M. National Survey of Midlife Development in the United States (MIDUS II): Biomarker Project, 2004-2009. Inter-university Consortium for Political and Social Research (ICPSR) [distributor]. 2013

38. Mattei J, Bhupathiraju S, Tucker KL. Higher adherence to a diet score based on American Heart Association recommendations is associated with lower odds of Allostatic load and metabolic 
syndrome in Puerto Rican adults. The Journal of Nutrition. 2013; 143:1753-1759. [PubMed: 24005611]

39. Mattei J, Demissie S, Falcon LM, Ordovas JM, Tucker K. Allostatic load is associated with chronic conditions in the Boston Puerto Rican health study. Soc Sci Med. 2010; 70:1988-1996. [PubMed: 20381934]

40. Mattei J, Noel SE, Tucker KL. A meat, processed meat, and French fries dietary pattern is associated with high allostatic load in Puerto Rican older adults. J Am Diet Assoc. 2011; 111:1498-1506. [PubMed: 21963016]

41. Fang J. Using SAS Procedures FREQ, GENMOD, LOGISTIC, and PHREG to Estimate Adjusted Relative Risks - A Case Study. SAS Global Forum 2011 Statistics and Data Analysis. 2011

42. Baron RM, Kenny DA. The moderator-mediator variable distinction in social psychological research: conceptual, strategic, and statistical considerations. J Pers Soc Psychol. 1986; 51:11731182. [PubMed: 3806354]

43. Pearl RL, Dovidio JF, Puhl RM. Visual portrayals of obesity in health media: promoting exercise without perpetuating weight bias. Health Educ Res. 2015; 30:580-590. [PubMed: 26116585]

44. Incollingo Rodriguez AC, Epel ES, White ML, et al. Hypothalamic-pituitary-adrenal axis dysregulation and cortisol activity in obesity: a systematic review. Psychoneuroendocrinology. 2015; 62:301-318. [PubMed: 26356039]

45. Barboza Solis C, Kelly-Irving M, Fantin R, et al. Adverse childhood experiences and physiological wear-and-tear in midlife: findings from the 1958 British birth cohort. Proc Natl Acad Sci. 2015; 112:E738-746. [PubMed: 25646470]

46. Wagner JA, Tennen H, Feinn R, Finan PH. Racial discrimination and metabolic control in women with type 2 diabetes. Ethn Dis. 2013; 23:421-427. [PubMed: 24392603]

47. Sutin AR, Stephan Y, Terracciano A. Weight discrimination and risk of mortality. Psychol Sci. 2015; 26:1803-1811. [PubMed: 26420442]

48. Gay JL, Salinas JJ, Buchner DM, et al. Meeting physical activity guidelines is associated with lower allostatic load and inflammation in Mexican Americans. J Immigr Minor Health. 2015; 17:574-581. [PubMed: 24242155]

49. Seeman TE, McEwen BS, Rowe JW, Singer BH. Allostatic load as a marker of cumulative biological risk: MacArthur studies of successful aging. Proc Natl Acad Sci. 2001; 98:4770-4775. [PubMed: 11287659] 


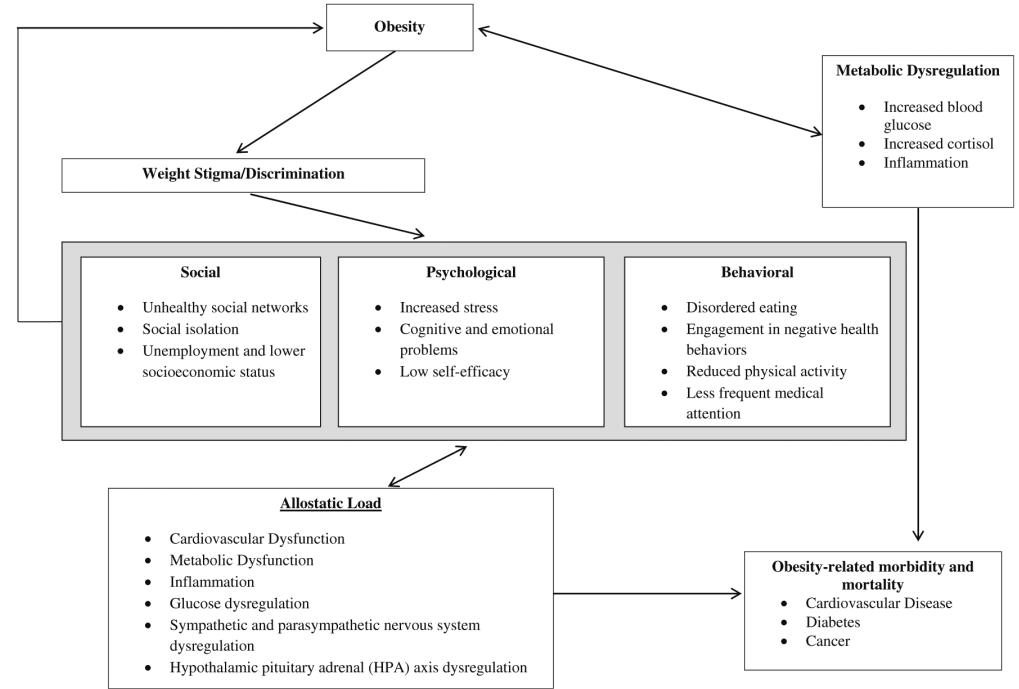

Fig. 1.

Conceptual model of potential pathways through which obesity and weight discrimination are associated with allostatic load (adapted from Gruenwald et al. [31] and Tomiyama et al. [3]) 


\section{Table 1}

Mean values and population-specific high-risk cut points for allostatic load parameters in the MIDUS Biomarker Substudy

\begin{tabular}{|c|c|c|c|c|c|c|}
\hline $\begin{array}{l}\text { System and representative } \\
\text { biomarkers }\end{array}$ & Number & Mean & SD & Min & $\operatorname{Max}$ & $\begin{array}{l}\text { High-risk cut point by } \\
\text { population-specific } \\
\text { quartile }\end{array}$ \\
\hline \multicolumn{7}{|l|}{ Cardiovascular } \\
\hline Resting SBP (mmHg) & 1254 & 131.5 & 18.3 & 83.0 & 222.0 & $\geq 144.0(n=309)$ \\
\hline Resting heart rate (bpm) & 1253 & 71.1 & 11.2 & 36.0 & 111.0 & $\geq 79.0(n=314)$ \\
\hline Resting pulse pressure (mmHg) & 1254 & 55.8 & 14.7 & 24.0 & 114.0 & $\succeq 65.0(n=312)$ \\
\hline \multicolumn{7}{|l|}{ Metabolic lipids } \\
\hline BMI $\left(\mathrm{kg} / \mathrm{m}^{2}\right)$ & 1254 & 29.8 & 6.63 & 15.0 & 65.1 & $233.1(n=313)$ \\
\hline WHR & 1253 & 0.89 & 0.10 & 0.62 & 1.72 & $\searrow 0.97(n=316)$ \\
\hline Triglycerides (mg/dL) & 1244 & 132.5 & 131.8 & 25.0 & 3299.0 & $\geq 156.0(n=312)$ \\
\hline HDL cholesterol (mg/dL) & 1242 & 55.4 & 18.0 & 19.0 & 121.0 & $\leq 42.0(n=310)$ \\
\hline LDL cholesterol (mg/dL) & 1242 & 105.5 & 35.4 & 6.00 & 283.0 & $\geq 128.0(n=310)$ \\
\hline \multicolumn{7}{|l|}{ Metabolic glucose metabolism } \\
\hline Glycosylated hemoglobin (\%) & 1235 & 6.10 & 1.16 & 3.58 & 19.7 & $ð 6.24(n=314)$ \\
\hline Fasting glucose $(\mathrm{mg} / \mathrm{dL})$ & 1236 & 102.1 & 28.4 & 5.00 & 418.0 & $\geq 105.0(n=314)$ \\
\hline Insulin resistance (HOMA-IR) & 1236 & 3.58 & 3.98 & 0.04 & 53.7 & $\geq 4.36(n=310)$ \\
\hline \multicolumn{7}{|l|}{ Inflammation } \\
\hline $\mathrm{CRP}(\mathrm{mg} / \mathrm{L})$ & 1235 & 3.02 & 4.78 & 0.14 & 61.7 & $23.66(n=309)$ \\
\hline IL6 (pg/mL) & 1243 & 3.04 & 3.04 & 0.16 & 23.0 & $23.48(n=310)$ \\
\hline Fibrinogen $(\mathrm{mg} / \mathrm{dL})$ & 1235 & 348.9 & 87.8 & 45.0 & 857.0 & $2399.0(n=313)$ \\
\hline sE-selectin (ng/MI) & 1242 & 43.4 & 22.7 & 0.09 & 178.1 & $251.9(n=310)$ \\
\hline sICAM-1 (ng/MI) & 1242 & 288.5 & 115.6 & 44.0 & 1076.6 & $\geq 335.8(n=310)$ \\
\hline \multicolumn{7}{|l|}{ Sympathetic nervous system } \\
\hline Urine epinephrine ( $\mu \mathrm{g} / \mathrm{g}$ creatine) & 1233 & 1.96 & 1.28 & 0.09 & 10.6 & $22.47(n=308)$ \\
\hline Urine norepinephrine ( $\mu \mathrm{g} / \mathrm{g}$ creatine) & 1243 & 27.4 & 13.9 & 3.50 & 187.1 & $233.0(n=311)$ \\
\hline \multicolumn{7}{|l|}{ Hypothalamic pituitary adrenal axis } \\
\hline Urine cortisol ( $\mu \mathrm{g} / \mathrm{g}$ creatine) & 1252 & 15.8 & 24.6 & 0.40 & 725.0 & $\geq 20.0(n=308)$ \\
\hline Blood DHEA-S $(\mu \mathrm{g} / \mathrm{dL})$ & 1239 & 105.1 & 77.0 & 0.90 & 685.0 & $51.0(n=313)$ \\
\hline \multicolumn{7}{|l|}{ Parasympathetic nervous system } \\
\hline SDRR (msec) & 1148 & 35.6 & 17.2 & 5.56 & 138.8 & 느.7 $(n=287)$ \\
\hline RMSSD & 1148 & 22.9 & 17.7 & 2.64 & 209.7 & $\unlhd 2.1(n=287)$ \\
\hline Low frequency spectral power & 1148 & 424.3 & 607.5 & 1.60 & $10,943.6$ & $\unlhd 14.6(n=287)$ \\
\hline High frequency spectral power & 1148 & 316.5 & 729.4 & 2.45 & $15,731.7$ & $58.8(n=287)$ \\
\hline Allostatic load & 1233 & 1.72 & 1.03 & 0 & 5.03 & \\
\hline Allostatic load (with medication data) & 1233 & 1.94 & 1.10 & 0 & 5.37 & \\
\hline
\end{tabular}

Allostatic Load was defined in accordance with previous studies conducted within this population using a score that captured dysregulation across seven systems, including multiple markers of cardiovascular pathways, sympathetic nervous system, parasympathetic nervous system, HPA axis, inflammation, lipid and general metabolic activity, and glucose metabolism, and could range from 0 to 7

$B M I$ body mass index, $C R P C$-reactive protein, $D H E A-S$ dehydroepiandrosterone sulfate, $P N S$ parasympathetic nervous system, $H P A$ hypothalamic pituitary axis, $I L-6$ interleukin-6, RMSSD root mean square of successive differences, $S B P$ systolic blood pressure, $S D R R$ the 
standard deviation of R-R (heartbeat to heartbeat) intervals, $s E$-selectin soluble adhesion molecule E-selectin, sICAM soluble intracellular adhesion molecule-1, SNS sympathetic nervous system 


\section{Table 2}

\section{Descriptive characteristics of the MIDUS participants at 10-year follow-up, $(n=932-1255)$}

\begin{tabular}{|c|c|}
\hline Age & $57.3(11.5)$ \\
\hline Sex (\% female) & 56.8 \\
\hline \multicolumn{2}{|l|}{ Race } \\
\hline White & 93.1 \\
\hline Black & 2.6 \\
\hline Other & 4.4 \\
\hline \multicolumn{2}{|l|}{ Educational attainment (\%) } \\
\hline Less than high school & 4.3 \\
\hline High school & 19.9 \\
\hline Some college & 29.2 \\
\hline College and above & 46.6 \\
\hline Household income (>\$100,000/year) & 21.4 \\
\hline Regular physical activity $(\%)^{a}$ & 76.5 \\
\hline \multicolumn{2}{|l|}{ Smoking status (\%) } \\
\hline Never & 52.4 \\
\hline Past & 32.6 \\
\hline Current & 14.9 \\
\hline Body mass index & $29.8(6.6)$ \\
\hline \multicolumn{2}{|l|}{ Weight category $(\%)$} \\
\hline Overweight & 35.1 \\
\hline Obesity & 41.2 \\
\hline \multicolumn{2}{|l|}{ Perceived weight discrimination $(\%)^{b}$} \\
\hline Baseline & 3.96 \\
\hline 10-year follow-up & 6.17 \\
\hline \multicolumn{2}{|l|}{ Perceived weight discrimination score ${ }^{b}$} \\
\hline Baseline & $0.13(0.76)$ \\
\hline 10-year follow-up & $0.22(1.09)$ \\
\hline High allostatic $\operatorname{load}^{c}$ & 18.3 \\
\hline High allostatic load (excluding medication) ${ }^{c}$ & 12.9 \\
\hline
\end{tabular}

Continuous variables are expressed as mean (SD) and categorical variables as percentages

a Physical activity was defined as the percentage of those who regularly exercised at least 20 min three times per week

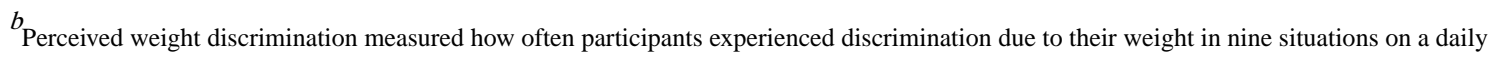
basis. For the categorical measure, anyone who reported any weight discrimination ("often" or "sometimes") was counted. For the continuous score measure, we summed the number of instances a person reported discrimination "sometimes" (assigned as 1 point) or "often" (assigned as 2 points). Individuals who reported discrimination "never" or "rarely" received a score of 0 . Baseline values were carried forward for individuals who reported weight discrimination at baseline, but had missing data at follow-up

CHigh allostatic load was defined as greater than or equal to 3 dysregu-lated systems and low allostatic load was defined as less than 3 . Allostatic load was measured at follow-up 
Table 3

Relative risk of high allostatic load based on perceived baseline and long-term weight discrimination in the MIDUS study

\begin{tabular}{|c|c|c|c|c|c|c|c|c|c|}
\hline & \multicolumn{3}{|c|}{ High allostatic load ${ }^{a}$} & \multicolumn{3}{|c|}{ Continuous allostatic load $b$} & \multicolumn{3}{|c|}{$\begin{array}{l}\text { High allostatic load } \\
\text { (excluding BMI and } \\
\text { WHR) }\end{array}$} \\
\hline & $\mathbf{R R}$ & $95 \% \mathrm{CI}$ & $p$ value & $\beta$ & SE & $p$ value & $\mathbf{R R}$ & $95 \% \mathrm{CI}$ & $p$ value \\
\hline \multicolumn{10}{|c|}{ Baseline perceived weight discrimination ${ }^{c}$} \\
\hline Age- and sex-adjusted & 2.60 & $1.60,4.23$ & 0.0001 & 0.15 & 0.04 & 0.0005 & 1.92 & $1.14,3.23$ & 0.01 \\
\hline Multivariable-adjusted model $1^{d}$ & 2.42 & $1.44,4.04$ & 0.0008 & 0.14 & 0.04 & 0.001 & 1.78 & $1.02,3.11$ & 0.04 \\
\hline Multivariable-adjusted model $2^{e}$ & 2.31 & $1.38,3.84$ & 0.001 & 0.13 & 0.04 & 0.002 & 1.71 & $0.99,2.97$ & 0.05 \\
\hline Multivariable-adjusted model $3^{f}$ & 2.23 & $1.28,3.87$ & 0.004 & 0.13 & 0.04 & 0.005 & 1.61 & $0.90,2.87$ & 0.11 \\
\hline Multivariable-adjusted model $4^{g}$ & 2.07 & $1.21,3.55$ & 0.008 & 0.11 & 0.04 & 0.01 & 1.55 & $0.87,2.75$ & 0.13 \\
\hline \multicolumn{10}{|c|}{ Long-term perceived weight discrimination $c, h$} \\
\hline Age- and sex-adjusted & 2.50 & $1.72,3.63$ & $<0.0001$ & 0.21 & 0.04 & $<0.0001$ & 1.87 & $1.25,2.79$ & 0.002 \\
\hline Multivariable-adjusted model $1^{d}$ & 2.47 & $1.65,3.69$ & $<0.0001$ & 0.21 & 0.04 & $<0.0001$ & 1.79 & $1.15,2.78$ & 0.01 \\
\hline Multivariable-adjusted model $2^{e}$ & 2.37 & $1.58,3.56$ & $<0.0001$ & 0.20 & 0.04 & $<0.0001$ & 1.73 & $1.11,2.69$ & 0.02 \\
\hline Multivariable-adjusted model $3^{f}$ & 2.27 & $1.45,3.56$ & 0.0003 & 0.21 & 0.05 & $<0.0001$ & 1.66 & $1.03,2.69$ & 0.04 \\
\hline Multivariable-adjusted model $4^{g}$ & 2.16 & $1.39,3.36$ & 0.0007 & 0.19 & 0.05 & 0.0001 & 1.62 & $1.01,2.62$ & 0.047 \\
\hline
\end{tabular}

${ }^{a}$ High allostatic load was defined as greater than or equal to three dysregulated systems, and low allostatic load was defined as less than three. Allostatic load was measured at follow-up, and medication usage was included in the definition

$b_{\text {Family status was added to the continuous models as a random effect }}$

$c$ Only individuals who reported discrimination "sometimes" or "often" were coded as having experienced discrimination

$d$ Model 1 includes age, sex, race (white, black, other), household income (>\$100,000/year), smoking status (never, former, current), educational attainment $(<$ high school, high school graduate, some college, college graduate or more)

$e_{\text {Model }} 2$ includes covariates in model 1 plus engagement in regular exercise at least 20 min three times per week

$f_{\text {Model }} 3$ includes covariates in models 1-2 plus perceived race, sex, and age discrimination at baseline

$g_{\text {Model } 4}$ includes covariates in models 1-3 plus baseline BMI

$h$ Long-term weight discrimination was computed as the average value of perceived discrimination at baseline and at 10 -year follow-up for those who had both measures. For individuals with no baseline measure, but with a measure at 10 years, long-term discrimination was computed as their reported discrimination at 10 years 


\section{Table 4}

Relative risk of high allostatic load system parameters based on perceived long-term weight discrimination in the MIDUS study

\begin{tabular}{|c|c|c|c|c|}
\hline & $\begin{array}{l}\text { Percent with } \\
\text { dysregulated } \\
\text { system }\end{array}$ & $\mathbf{R R}$ & $95 \% \mathrm{CI}$ & $p$ value \\
\hline Cardiovascular & 38.6 & & & \\
\hline Age and sex-adjusted & & 1.37 & $1.03,1.81$ & 0.03 \\
\hline Model $1^{a}$ & & 1.26 & $0.92,1.72$ & 0.15 \\
\hline Model $2^{b}$ & & 1.20 & $0.89,1.65$ & 0.23 \\
\hline Model $3^{c}$ & & 1.24 & $0.89,1.72$ & 0.20 \\
\hline Model $4^{d}$ & & 1.23 & $0.89,1.72$ & 0.21 \\
\hline Metabolic & 18.3 & & & \\
\hline Age and sex-adjusted & & 2.07 & $1.49,2.88$ & $<0.0001$ \\
\hline Model $1^{a}$ & & 2.07 & $1.45,2.97$ & $<0.0001$ \\
\hline Model $2^{b}$ & & 1.96 & $1.35,2.82$ & 0.0003 \\
\hline Model $3^{c}$ & & 1.66 & $1.07,2.57$ & 0.02 \\
\hline Model $4^{d}$ & & 1.56 & $1.02,2.40$ & 0.04 \\
\hline Glucose metabolism & 23.5 & & & \\
\hline Age and sex-adjusted & & 2.29 & $1.62,3.24$ & $<0.0001$ \\
\hline Model $1^{a}$ & & 2.29 & $1.43,3.67$ & 0.0006 \\
\hline Model $2^{b}$ & & 2.26 & $1.59,3.22$ & $<0.0001$ \\
\hline Model $3^{c}$ & & 2.10 & $1.40,3.15$ & 0.0003 \\
\hline Model $4^{d}$ & & 1.99 & $1.34,2.95$ & 0.0006 \\
\hline Inflammation & 26.6 & & & \\
\hline Age and sex-adjusted & & 1.89 & $1.37,2.61$ & $<0.0001$ \\
\hline Model $1^{a}$ & & 1.99 & $1.44,2.76$ & $<0.0001$ \\
\hline Model $2^{b}$ & & 1.91 & $1.37,2.67$ & 0.0001 \\
\hline Model $3^{c}$ & & 1.83 & $1.27,2.64$ & 0.001 \\
\hline Model $4^{d}$ & & 1.76 & $1.22,2.54$ & 0.003 \\
\hline Sympathetic nervous system ${ }^{e}$ & 12.4 & & & \\
\hline Age and sex-adjusted & & 1.27 & $0.70,2.32$ & 0.43 \\
\hline Model $1^{a}$ & & 1.26 & $0.67,2.35$ & 0.47 \\
\hline Model $2^{b}$ & & 1.24 & $0.66,2.32$ & 0.50 \\
\hline Model $3^{c}$ & & 1.31 & $0.64,2.68$ & 0.46 \\
\hline Model $4^{d}$ & & 1.44 & $0.70,2.96$ & 0.32 \\
\hline Hypothalamic pituitary axis & 43.6 & & & \\
\hline
\end{tabular}




\begin{tabular}{|c|c|c|c|c|}
\hline & $\begin{array}{l}\text { Percent with } \\
\text { dysregulated } \\
\text { system }\end{array}$ & $\mathbf{R R}$ & $95 \% \mathrm{CI}$ & $p$ value \\
\hline Age and sex-adjusted & & 0.94 & $0.71,1.24$ & 0.66 \\
\hline Model $1^{a}$ & & 0.96 & $0.71,1.28$ & 0.77 \\
\hline Model $2^{b}$ & & 0.97 & $0.72,1.30$ & 0.84 \\
\hline Model $3^{c}$ & & 0.94 & $0.69,1.30$ & 0.72 \\
\hline Model $4^{d}$ & & 0.95 & $0.69,1.31$ & 0.77 \\
\hline $\begin{array}{l}\text { Parasympathetic nervous } \\
\text { system }^{e}\end{array}$ & 19.6 & & & \\
\hline Age and sex-adjusted & & 1.42 & $0.90,2.23$ & 0.13 \\
\hline Model $1^{a}$ & & 1.42 & $0.86,2.33$ & 0.17 \\
\hline Model $2^{b}$ & & 1.38 & $0.83,2.29$ & 0.21 \\
\hline Model $3^{c}$ & & 1.28 & $0.75,2.21$ & 0.36 \\
\hline Model $4^{d}$ & & 1.29 & $0.75,2.21$ & 0.37 \\
\hline
\end{tabular}

Long-term perceived weight discrimination represents the average value of perceived discrimination at baseline and at follow-up for those who had both measures. For individuals with only one measure, long-term discrimination represents their reported discrimination at that time point

a Model 1 includes age, sex, race (white, black, other), household income (>\$100,000/year), smoking status (never, former, current), educational attainment (< high school, high school graduate, some college, college graduate or more)

$b_{\text {Model }} 2$ includes covariates in model 1 plus engagement in regular exercise at least 20 min three times per week

$c_{\text {Model }} 3$ includes covariates in models 1-2 plus perceived race, sex, and age discrimination at baseline

${ }^{d}$ Model 4 includes covariates in models 1-3 plus baseline BMI

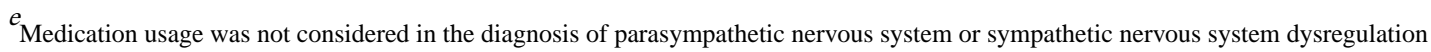

